This is the accepted version of the article:

Amadei C.A., Lai C.-Y., Esplandiu M.J., Alzina F., Vecitis C.D., Verdaguer A., Chiesa M.. Elucidation of the wettability of graphene through a multi-length-scale investigation approach. RSC Advances, (2015). 5. : 39532 - . 10.1039/c5ra04397b.

Available at: https://dx.doi.org/10.1039/c5ra04397b 


\title{
Elucidation on the wettability of graphene throughout a multi-length-scale investigation approach
}

\author{
Carlo A. Amadei ${ }^{a, b}$, Chia-Yun Lai ${ }^{b}$, Maria Jose Esplandiu ${ }^{a}$, Francesc Alzina $^{a}$, Chad D. \\ Vecitis $^{c}$, Albert Verdaguer ${ }^{a}$, Matteo Chiesa ${ }^{b, *}$
}

${ }^{a}$ ICN2 - Institut Catala de Nanociencia i Nanotecnologia, and CSIC - Consejo Superior de Investigaciones Cientificas. ICN2 Building, Campus UAB, 08193 Bellaterra (Barcelona), Spain
${ }^{\mathrm{b}}$ Laboratory for Energy and NanoScience (LENS), Institute Center for Future Energy (iFES), Masdar Institute of Science and Technology, Abu Dhabi, UAE
${ }^{\mathrm{c}}$ School of Engineering and Applied Sciences, Harvard University, Cambridge, MA, United States 02138.

*Corresponding author: mchiesa@masdar.ac.ae
Present address: PO BOX 54224 Masdar Institute, Abu Dhabi, UAE 


\begin{abstract}
Univocal conclusions around the wettability of graphene exposed to environmental conditions remain elusive despite the recent efforts of several research groups. The main discrepancy rests on the question whether a graphene monolayer (GML) is transparent or not to water and more generally what is the role that the substrate plays in determining the degree of wetting of the GML. In this work, we investigate the water transparency of GML by means of a multi-length-scale approach. We complement traditional static contact angle measurements and environmental scanning electron microscope experiments with atomic force microscopy based force spectroscopy to assess the role that intermolecular interactions play in determining the wetting of GML. To gain deeper insight on the wetting transparency issue, we perform experiments on inert metals, such as gold and platinum, covered or not by GML. The comparison of the results obtained for different systems (i.e. GML covered and uncovered inert metals), provides unambiguous evidence that support the non-wetting transparency theory of GML. This work aims to assist the development of technologies based on graphene/water interaction, such as graphitic membranes for water separation processes.
\end{abstract}




\section{Introduction}

Graphene is a single layer $\mathrm{sp}^{2}$ lattice of carbon atoms, organized in a honeycomb atomic configuration. Since its first isolation by Novoselov and Geim in 2004, ${ }^{1}$ graphene has attracted the interest of the scientific community which investigated the properties of this fascinating $2 \mathrm{D}$ material system. ${ }^{2}$ The superior properties of graphene span from electrical ${ }^{3}$ and thermal $^{4}$ conductivity to mechanical stiffness, ${ }^{5}$ elasticity ${ }^{6}$ and optical transparency. ${ }^{7}$ It is the combination of such properties that may lead graphene to replace other materials in existing applications ${ }^{8}$ or, more importantly, could trigger the fantasy of researchers in the conception of new applications. ${ }^{9}$ As a 2D material, graphene's surface chemistry is an important aspect to evaluate how graphene interacts with its surrounding. The latter can be represented by water or air, in applications for which graphene is implemented as ion-filtration membrane, ${ }^{10,11}$ gas barrier respectively ${ }^{12,13,14}$ or self-cleaning coating. ${ }^{15,16}$ Alternatively, the surroundings can be also represented by the human organism, if graphene is used in biomedical applications, such as tissue engineering ${ }^{17}$ or drug delivery, ${ }^{18}$ as promising results demonstrated. ${ }^{19}$ For all these possible applications carefully determining the interaction between graphene and its environment ${ }^{20}$ will assist researchers in tailoring and optimizing the functionality of graphene-based applications.

In this context, graphene/water is one of the most fundamental interactions and it has been recently investigated by several research groups. However, a controversial scenario emerges from these studies, which can be summarized with the following question: "Is a monolayer of graphene transparent to water-substrate interactions or not?" Rafiee et al. ${ }^{21}$ stated that a graphene monolayer (GML) on top of a metal substrate is wetting transparent to long-range (i.e. up to $5 \mathrm{~nm}$ ) van der Waals (vdW) forces, whereas the transparency is lost, if the substrate forms short-range 
chemical bonds (i.e. H-bond) with water. By contrast, Raj et al. ${ }^{22}$ rejected the graphene wetting transparency theory demonstrating that the type of interaction and the role of the substrate is negligible. A further model was proposed by Shih et al. ${ }^{23}$ suggesting that graphene wetting transparency depends on the wettability of the substrate. Recently, Li et al. ${ }^{24}$ reported a transition in the wettability of graphene from hydrophilic to hydrophobic behaviour after exposed to the ambient air. The cause of this transition is explained with the adsorption of hydrocarbon contaminants on graphene surface, ${ }^{25}$ which echoes earlier investigations on the wettability transition of graphitic surfaces. ${ }^{26}$ The adsorption of molecules from the atmosphere, demonstrated on graphitic surfaces ${ }^{27}$ by means of atomic force microscope (AFM) and spectroscopy techniques, ${ }^{28,29}$ can represent the root of the debate around the wettability of graphene. Research groups may have analysed the same graphene system, but decorated with different adsorbed molecules, and this might have led to different experimental results, such as contact angle measurements. Furthermore, the discrepancy in the literature extends also to modelling efforts, such as molecular dynamics (MD) simulations. Disagreement between theoretical simulations can be attributed to the different water-carbon interaction model employed $^{30}$ or different input parameters. For instance the cut-off distance used by Shih et al. (i.e. $0.9 \mathrm{~nm}$ ) is less than half compared to the one used by Raj et al. (i.e. $2 \mathrm{~nm}$ ). This highlights the need for nanoscale measurements, which can shed light on the fundamental interaction between water and graphene-based systems. ${ }^{31}$ This work aims to study the wettability of graphene from macro- to nano-scale dimensions. The investigation is carried out on surfaces after being exposed for days to environmental conditions in order to study stable systems, which are not subjected to time-dependent wettability. ${ }^{24,25}$ Moreover, exposed surfaces can represent models samples, when graphene will be applied in applications under ambient conditions. Traditional contact angle 
studies are employed to examine the macroscopic wettability of graphene. Environmental scanning electron microscope (ESEM) allows imaging droplets of circa $10 \mu \mathrm{m}$, accessing to the micron-range-wettability. Finally, by means of AFM we reconstruct the force profile between the tip and the substrate, ${ }^{32}$ evaluating the nanoscale interaction. All our experiments are carried out for a GML grown via chemical vapor deposition (CVD) on platinum (Pt) and GML mechanically transferred onto gold $(\mathrm{Au})$ foil (see Electronic Supplementary Information, ESI). The results are then compared with those obtained on the bare substrates (i.e. Pt and Au). Pt and Au are chosen because of their lowest reactivity towards water and its constituting elements among metals ${ }^{33,34}$. This yields Pt and Au resistance to corrosion and oxidation. Despite their possible hydrocarbon contaminations, ${ }^{35}$ the lowest reactivity of $\mathrm{Pt}$ and $\mathrm{Au}$ allows these metal surfaces to interact with water via vdW interactions something that makes the comparison between the GML/metal and bare metal significant for evaluating the wetting transparency of graphene. In case of comparable wettability results for GML/metal surface and bare metal at different length scale, one would confirm wetting transparency of graphene. On the other hand, incompatible wettability results between the two systems would lead to the rejection of wetting transparency theory.

\section{Experiemental}

Graphene synthesis and characterization: graphene films were grown on Pt foils $(0.025 \mathrm{~mm}$ thick, Premion, 99.99\%, Alfa-Aesar) with low pressure chemical vapor deposition using a Lindberg blue tube furnace. The Pt foil was loaded in the furnace quartz tube followed by air evacuation up to reach a basal pressure of 2 mbar. Then a flux of Ar of $400 \mathrm{sccm}$ was introduced in the furnace tube while heating up to $1020{ }^{\circ} \mathrm{C}$. Prior to the graphene growth the sample was annealed at that temperature in presence of $400 \mathrm{sccm}$ of $\mathrm{Ar}$ and $20 \mathrm{sccm}$ of $\mathrm{H}_{2}$ during 30 minutes. 
After that the graphene growth was taking place by introducing $35 \mathrm{sccm}$ of $\mathrm{CH}_{4}$ to the previous fluxes of $\mathrm{Ar}$ and $\mathrm{H}_{2}$ during 10 minutes and at the same temperature. Then the sample was fast cooling under the flow of $\mathrm{Ar}$ and $\mathrm{H}_{2}$. The GML/Pt was purchased from Graphenea, which produced the sample according to the following procedure. The sample was prepared by growing the GML on $\mathrm{Cu}$ foil. Then, a wet transfer process occurred in order to deposit the graphene film on the new substrate $(\mathrm{Au})$. During the process, protective polymer (PMMA) was used. MicroRaman spectra were taken in backscattering geometry at room temperature using the $5145 \AA$ line of an Argon ${ }^{+}$laser with a Jobin-Yvon T-64000 spectrometer in single mode configuration and liquid-nitrogen-cooled CCD detection. The laser spot size was $\sim 1 \mu \mathrm{m}$ and the laser power onto the sample was kept below $0.5 \mathrm{~mW}$ to avoid sample overheating.

Atomic force microscope force spectroscopy: most of the information regarding material properties can be extracted from the force sensed by the probe when approaching the sample. The ability of recovering the force versus $d$ from observables is defined as AFM force spectroscopy. In our investigation the AFM is operating in AM and the observable used for the force spectroscopy are the oscillation amplitude (A) and phase shift ( $\Phi$ ) relative to the driving force. By means of these observables we can recover the interactions between the tip and the sample, which are generally divided between conservative and dissipative interactions. For the reconstruction of the conservative part of the force, we exploited Sader-Jarvis-Katan (SJK) formalism (Eq.1), ${ }^{36}$ which represents the evolution of the earlier formalisms proposed by Giessibl $^{37}$ and $\operatorname{Sader}^{38}$ in frequency modulation (FM) AFM:

$$
F_{t s}(d)=2 k \int_{u=d}^{u=\infty}\left[\left(1+\frac{A^{1 / 2}(u)}{8 \sqrt{\pi(u-d)}}\right) \Omega(u)-\frac{A^{3 / 2}(u)}{\sqrt{2(u-d)}} \frac{d \Omega(u)}{d u}\right] d u
$$


where $\Omega$ is the normalized frequency shift expressed by:

$$
\Omega(d)=\left[1+\frac{A_{0}}{Q A} \cos (\Phi(d))\right]^{\frac{1}{2}}-1
$$

where $Q$ is the quality factor which has been quantified by performing the standard thermal analysis a tip-sample separation of $\sim 50 \mathrm{~nm}$. All the experiments are carried out with a Cypher AFM from Asylum Research and standard OLYMPUS cantilevers (AC160TS). The tip radius R was constantly monitored in situ to assure that no change occurs throughout the experiment. The monitoring of the tip is paramount, since it is well-known that $\mathrm{R}$ significantly affects the interaction force between the tip and the surface. ${ }^{39}$

The free amplitudes employed in these experiments were $A_{0} \approx 40 \mathrm{~nm}$. These relatively large values of free amplitude implied that smooth transitions from the attractive to the repulsive regime followed throughout this work. ${ }^{32}$ Thus, no transients due to discontinuous transitions were present in the interaction ${ }^{40}$ and the relevant attractive to repulsive distances were recovered successfully without discontinuities.

The dissipative interactions are evaluated with Cleveland's equation: ${ }^{41}$

$$
E_{\text {dis }}=\frac{\pi k A_{0} A}{Q}\left[\sin (\Phi)-\frac{A}{A_{0}}\right]
$$

Where all the parameters have been already described.

Contact angle measurements: macroscopic contact angle measurements were executed with a Krüss FM40Mk2 EasyDrop contact angle instrument. The standard software supplied by Krüss was used for the evaluation of the data. SCAs were measured using $2 \mu \mathrm{L}$ droplets and the data 
for each time step refers to 5 measurements. In order to determine the contact angle at the microscale, we employed an ESEM using FEI, Quanta 250 to investigate the surface wettability of the graphene surfaces. We achieved a static contact angle inside the ESEM (SCAESEM) chamber by carefully tuned the chamber pressure so that the droplets were condensed in a really slow manner (i.e. equilibrium) That is, we first cooled down the sample to $0^{\circ} \mathrm{C}$ while kept the chamber pressure at $100 \mathrm{~Pa}$. Then we slowly increased the chamber pressure to $580 \mathrm{~Pa}$ and waited the system to be in equilibrium (judged by the scanning images). Finally, the chamber pressure was slowly increased to $620 \mathrm{~Pa}$ and the droplets were condensed. The sample stage was tilted for 7$10^{\circ}$ in order to increase the droplets counts. Video was recorded while droplets were forming on the graphene surfaces. The droplets shape was then analyzed by ImageJ to obtain values of SCA at the micro scale.

\section{Results and discussion}

Graphene characterization. An extensive AFM study is carried out to characterize the GML/metal surface, however for clarity, the most representative data sets are reported in Figure 1. Figure $1 \mathrm{a}$ and $1 \mathrm{~b}$ represent topographical images acquired via amplitude modulation (AM) for GML on Pt and on Au, respectively. In order not to perturb the system, the tip was not in contact with the surface during the acquisition and the topographical image is dictated by long range interactions (i.e. vdW). The systems are characterized by similar roughness (i.e. topography RMS is $2.4 \mathrm{~nm}$ and $3.5 \mathrm{~nm}$ for GML/Pt and GML/Au, respectively) and they present sharply corrugations that can be connected to different interfacial restructuring during the graphene growth by CVD. ${ }^{42,43}$ The roughness values are comparable to the one obtained on the bare metals surfaces (see ESI Figure S1), thus the GML does not change the topography of the samples. 
Furthermore, the surfaces are clean at the micro-scale, avoiding influence of macrocontamination in the following studies of wettability. An intensity ratio of the Raman $2 \mathrm{D}$ and $\mathrm{G}$ peaks of $\mathrm{I}(2 \mathrm{D}) / \mathrm{I}(\mathrm{G})>3$, together with a full width at half-maximum (FWHM) of the 2D band of $\sim 35 \mathrm{~cm}^{-1}$ on the Raman spectra of, both, Pt and Au systems (Figure 1c and 1d) reveal the presence of monolayer graphene. $^{44,45}$

(a)

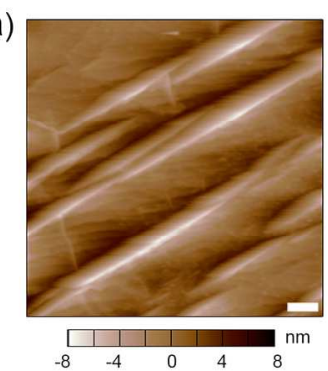

(c)

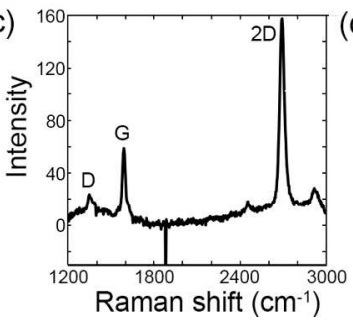

(b)

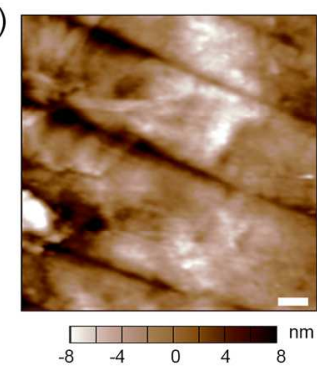

(d)

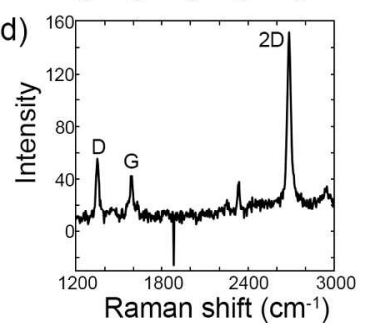

(e)

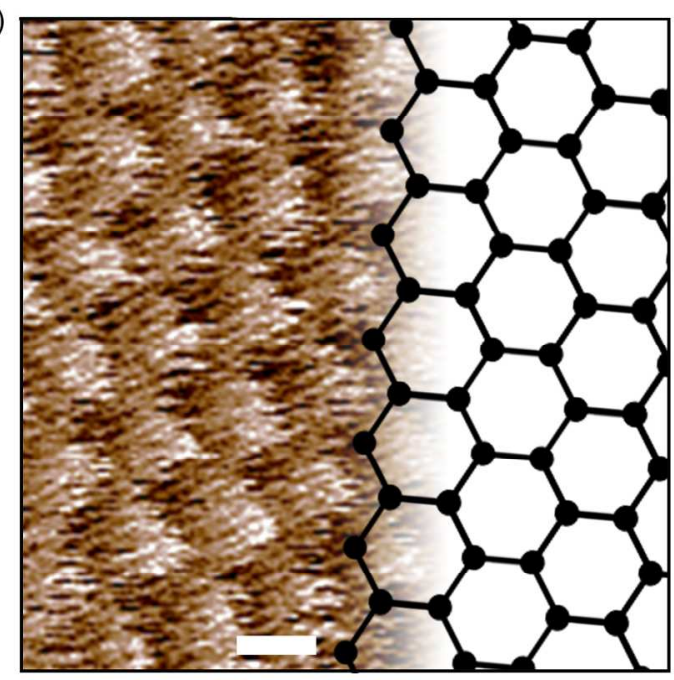

Figure 1: GML characterization by means of AFM and Raman spectroscopy. (a, b) AFM images acquired in non-contact mode on bare Pt and Au. Scale bars are $100 \mathrm{~nm}$. (c, d) Raman spectra of GML/Pt and GML/Au respectively. (e) AFM lateral force map acquired on GML/Au. Scale bar is 0.5 nm.

The presence of graphene on the surface is corroborated by a lateral force map (Figure 1e) obtained on GML/Au. In this mode of operation, the tip is in continuous contact with the surface and the force (i.e. lateral) experienced by the tip when scanning the sample is recorded. This force is mainly due to the local topography and it allows resolving the hexagonal pattern typical of the graphene. ${ }^{42}$ However, the lattice dimension is larger than $\mathrm{C}-\mathrm{C}$ bond and is due to the thermal drifting during the image acquisition. Nevertheless, this represents an important 
achievement, knowing the fact that the images are acquired in normal ambient conditions without additional sample preparation. A fast Fourier transform (FFT) of the hexagonal pattern is carried out in order to evaluate the crystallographic structure of the surface. Results show that the FFT is identical to the ones obtained by scanning tunneling microscope images or transmission electron microscope images ${ }^{46}$ (see ESI Figure S2).

Wettability analysis. First, we study the macroscopic wettability of the GML/metal and bare metal for the $\mathrm{Au}$ and $\mathrm{Pt}$ system (Figure 2). From Figure 2a one can observe a significant difference between the water static contact angle (SCA) for GML/Au and bare Au. In particular, the SCA of GML/Au is $\approx 93^{\circ}$, which is a value $10^{\circ}$ higher compared to the one obtained on bare Au. A similar trend was also observed by Li et $a l .{ }^{24}$ when analyzing GML/Au systems. In Figure $2 \mathrm{~b}$ the relative behaviour between the bare $\mathrm{Pt}$ and the Pt covered by GML is even more evident. To our knowledge, this is the first time that the SCA for GML/Pt has been reported. Pt surface is characterized by SCA values of $\approx 65^{\circ}$, whereas GML/Pt reaches value comparable to exposed highly ordered pyrolytic graphite (HOPG) ${ }^{25}$ and GML/Au. This highlights the low influence of the metal substrate in dictating the interaction with water. Note that during SCA image acquisition, no hysteresis of the contact line (solid/liquid/vapour) is observed, indicating that the system is in thermodynamic equilibrium with the surroundings. However, the results refer to metals exposed to environmental conditions and they could be subjected to molecular adsorption. We also perform SCA on bare metal surface after annealing process (see ESI). Those surface are characterized by SCA generally smaller than the ones of exposed surfaces, strengthening our observation on the low influence of the metal substrate. Fourier transform infrared spectroscopy confirms small water adsorption from the atmosphere (i.e. $14 \%$ of increase in the spectra 
intensity) as the metal surface has been exposed to ambient conditions. ESI also contains dynamic contact angel analysis performed on both bare metal and graphene covers systems.
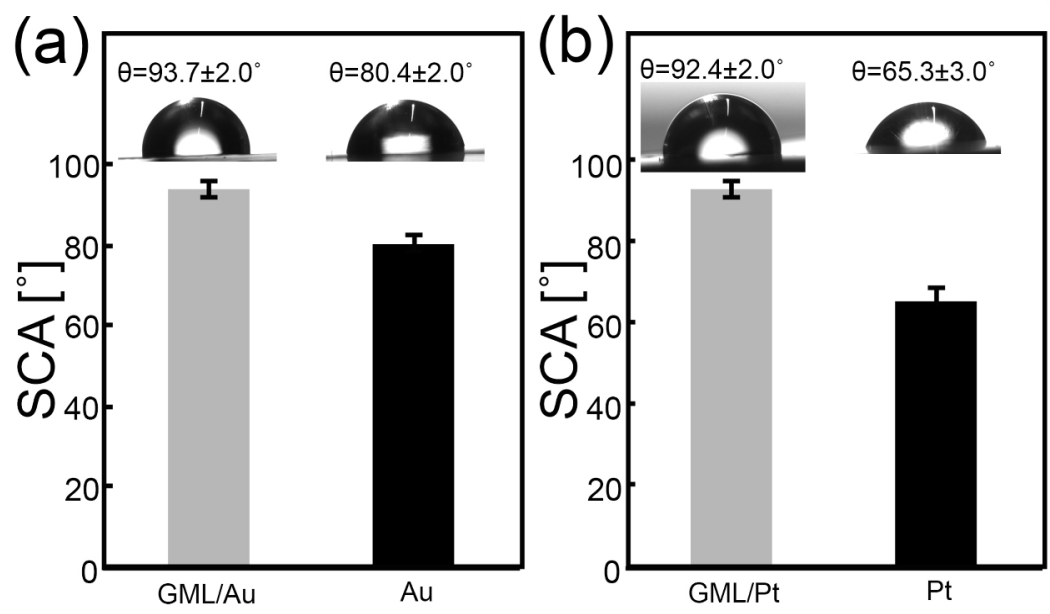

Figure 2: SCA analysis. (a) SCA on GML on Au and bare Au. (b) SCA on GML on Pt and bare Pt. Images illustrate the most representative contact angle for each case.

From a macroscopic point of view we moved our investigation towards the micron-range domain. With the use of ESEM, it is possible to describe the wetting behavior of the surface at the microscale, by analyzing the water droplets condensed on the surface. The implementation of ESEM in analyzing graphene wettability was already reported by Raj et al. ${ }^{22}$ with satisfactory results. Figure 3a represents a typical image obtained inside the ESEM chamber where all the droplets' SCA $_{\text {ESEM }}$ (ESEM subscript is used to distinguish between SCA obtained by traditional techniques) are evaluated and they represent the statistical population for that particular system. In Figure $3 b$ we display the most representative droplets for the four systems (i.e. GML/metal and bare metal for the two metals) and in Table 1 the statistical analysis (i.e. average and standard deviations of $\left.\mathrm{SCA}_{\mathrm{ESEM}}\right)$ is reported. The results are qualitatively in agreement with macroscopic results and they share three aspects with the macroscopic SCA. i) Surfaces of the metal covered by GML are more hydrophobic than the bare metal. In particular, $\mathrm{SCA}_{\mathrm{ESEM}}$ for 
$\mathrm{GML} / \mathrm{Au}$ and $\mathrm{GML} / \mathrm{Pt}$ is $\approx 15^{\circ}$ and $\approx 25^{\circ}$ higher compared to the bare Au and Pt respectively, which underlines the importance of GML in varying the surface wettability. ii) GML covered systems are characterized by comparable $\operatorname{SCA}_{\mathrm{ESEM}}$ (i.e. $75^{\circ}-79^{\circ}$ ), thus implying the minimal effect of the underneath substrate. iii) Quantitatively SCAESEM display lower values compare to traditional SCA, with a reduction in the order of $15-20 \%$. This difference could be attributed to the fact that the ESEM stage is tilted and, thus, the observation angle is not parallel to the surface $^{47}$ or to the different ESEM-chamber pressure (see ESI) compared to environmental pressure. $^{48}$
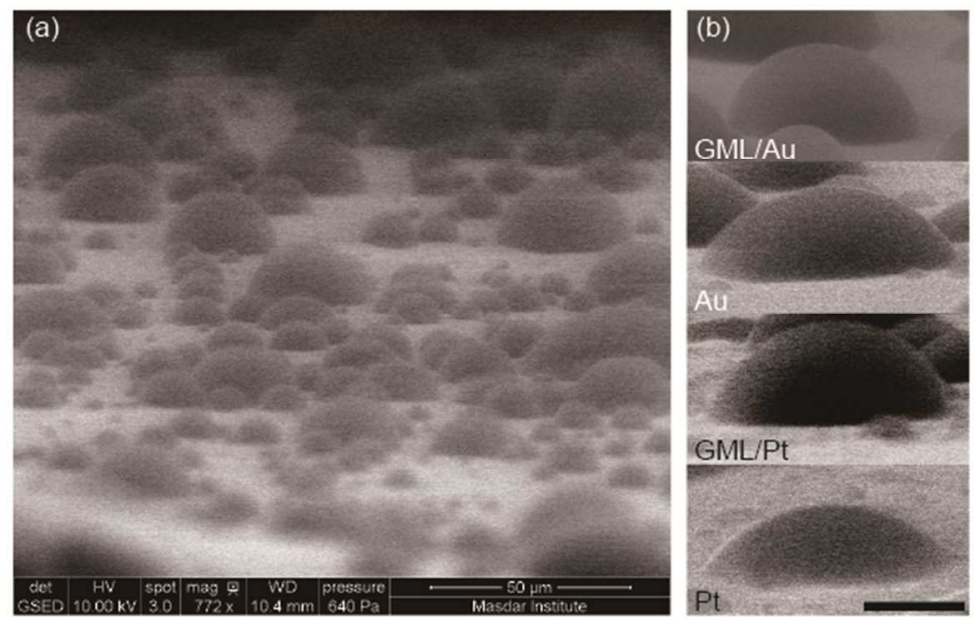

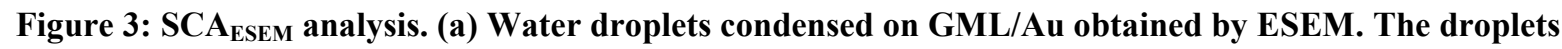
construct the statistics of Table 1. (b) Single zoom of a droplet formation for the four systems (GML/Au, bare $\mathrm{Au}, \mathrm{GML} / \mathrm{Pt}$ and bare Pt). Scale bar is $50 \mu \mathrm{m}$ and $5 \mu \mathrm{m}$ for (a) and (b) respectively.

Table 1: SCA $A_{\text {ESEM }}$ obtained for the four systems. Mean and standard deviation values refer to 15 measurements.

\begin{tabular}{|c|c|}
\hline System & $\operatorname{SCA}_{\text {ESEM }}\left(^{\circ}\right)$ \\
\hline GML/Au & $79.4 \pm 5.6$ \\
\hline $\mathrm{Au}$ & $64 \pm 5.4$ \\
\hline $\mathrm{GML} / \mathrm{Pt}$ & $75.9 \pm 5.1$ \\
\hline $\mathrm{Pt}$ & $51.9 \pm 4.6$ \\
\hline
\end{tabular}


Moved by the need of the research community to explore the wettability at smaller length scales, ${ }^{31}$ we proceed toward investigating the phenomena at the nanoscale length. Physically, we achieved this by exploiting a recent developed AFM force spectroscopy technique. The technique allows us to recover the conservative force field ${ }^{49}$ versus tip-sample minimum distance of approach (d) felt by the tip when interacting with the sample. The force field, $F_{t s}$, is then characterized by the distance of force of adhesion $\left(\Delta \mathrm{dF}_{\mathrm{AD}}\right.$, gray dash lines in Figure $\left.4 \mathrm{a}\right)$, which is a metric, defined as the range of horizontal distances for which $F_{t s}=C_{1} F_{A D}$; where $C_{1}$ is an arbitrary coefficient and $\mathrm{F}_{\mathrm{AD}}$ is the minima in the force field. In order to describe the force field in a complete manner, we carried out our analysis using seven different coefficients (i.e. 0.05, $0.1,0.2,0.35,0.5,0.65$ and 0.8$)$. In Figure $4 \mathrm{~b}$ and $4 \mathrm{c}$ the most representative normalized force fields (i.e. $\mathrm{F}_{\mathrm{ts}}{ }^{*}$ ) for the $\mathrm{Au}$ systems (i.e. GML/Au and bare $\mathrm{Au}$ ) and Pt system (GML/Pt and bare Pt) are reported. From these experimental plots, for the two graphene systems, the interaction of the GML/metal (gray lines) is shorter compared to the bare metal (black lines). For each sample, 50 force curves were randomly taken on the surface and the results are summarized in Figure $4 \mathrm{~d}$ and $4 \mathrm{e}$. In Figure $4 \mathrm{~d}$, it is possible to observe that $\Delta \mathrm{dF}_{\mathrm{AD}}$ for bare $\mathrm{Au}$ (black line) is higher compared to GML/Au (gray line) for all the seven $\mathrm{C}_{1}$ coefficients used. This means that the bare metal is characterized by an interaction with the tip at larger distances as compared to the one of the GML/Au surface. From Figure 4e, similar behaviour is obtained when the tip is interacting with the Pt system. Also in this case, the bare metal substrate (black line) interacts with the tip at larger distances as compared to the case of GML/Pt system. Quantitatively, the presence of the graphene reduces the distance of interaction of circa $20 \%$. For instance, in the case of Au, the presence of the graphene determines a decrease of $\Delta \mathrm{dF}_{\mathrm{AD}}$ from $\approx 0.29 \AA$ to $\approx 0.23 \AA$ and from 
$\approx 0.14 \AA$ to $\approx 0.10 \AA$ for $\mathrm{C}_{1}=0.05$ and $\mathrm{C}_{1}=0.8$ respectively. One could note that the tip interaction with the graphene covered systems present different shapes. This might be due to the fact that graphene systems are prepared with different fabrication methods (i.e. CVD and wet mechanical transfer) or to possible evolution of the graphene surface when exposed to environmental conditions. ${ }^{50}$
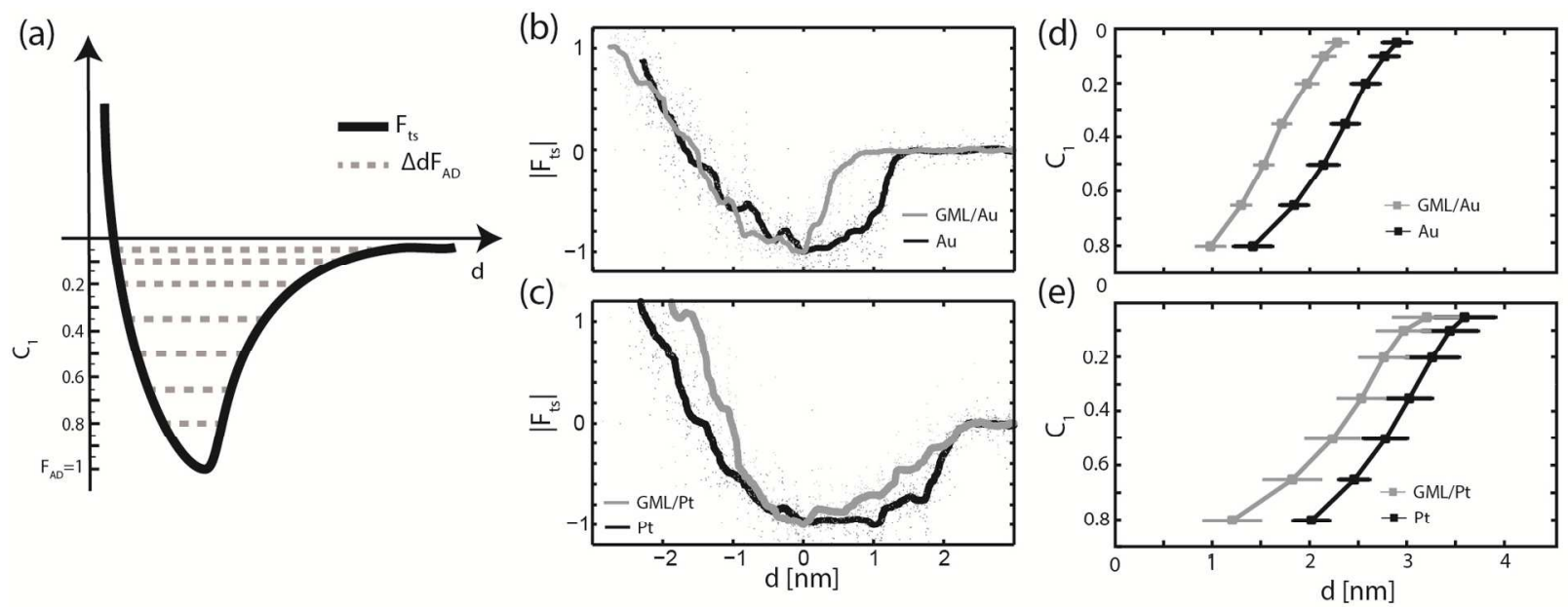

Figure 4: tip/surface conservative interactions by means of AFM. (a) Characterization of the conservative force field $\left(F_{t s}\right.$, black line) by means of $\Delta \mathrm{dF}_{\mathrm{AD}}$ (with gray dash lines) for different $\mathrm{C}_{1}$ coefficients. (b, c) Conservative interactions analyzed with $\Delta \mathrm{dF}_{\mathrm{AD}}$ for 7 different $\mathrm{C}_{1}$ coefficients $(0.05,0.1,0.2,0.35,0.5,0.65$ and 0.8) data refer to GML/Au-bare Au and GML/Pt-bare Pt. (d, e) Reconstructed conservative profiles interaction for GML/metal (gray lines) and bare metal (black lines).

We also analysed the nanoscale dissipative interactions, calculating the energy dissipation $\left(\mathrm{E}_{\mathrm{dis}}\right)$ between the oscillating tip and the surface, with the use of the Cleveland equation ${ }^{41}$ (see ESI). From Figure $5 \mathrm{a}$ it is possible to see that the bare metals are characterized by higher values of $\mathrm{E}_{\mathrm{dis}}$ compared to the metals covered by GML. The increase is on the order of 10-15 eV, which can be related to additional dissipative mechanisms (i.e. capillary phenomena) ${ }^{51}$ detected by the reconstructed energy dissipation profiles. In Figure $5 \mathrm{~b}$ we report the most representative normalized energy dissipation profile $\left(\mathrm{E}_{\mathrm{dis}}{ }^{*}\right)$ for the GML/Pt and bare Pt system. The bare metal surface is characterized with an energy dissipation profile which starts at larger distance 
compared to the graphene covered system, in accordance with the conservative interactions. This phenomenon, in combination with the presence of inflection points (red circles), is related to capillary interaction between the tip and the surface. Note that in ambient conditions AFM tips are usually covered by a thin layer of water (i.e. less than $1 \mathrm{~nm}) .^{51}$
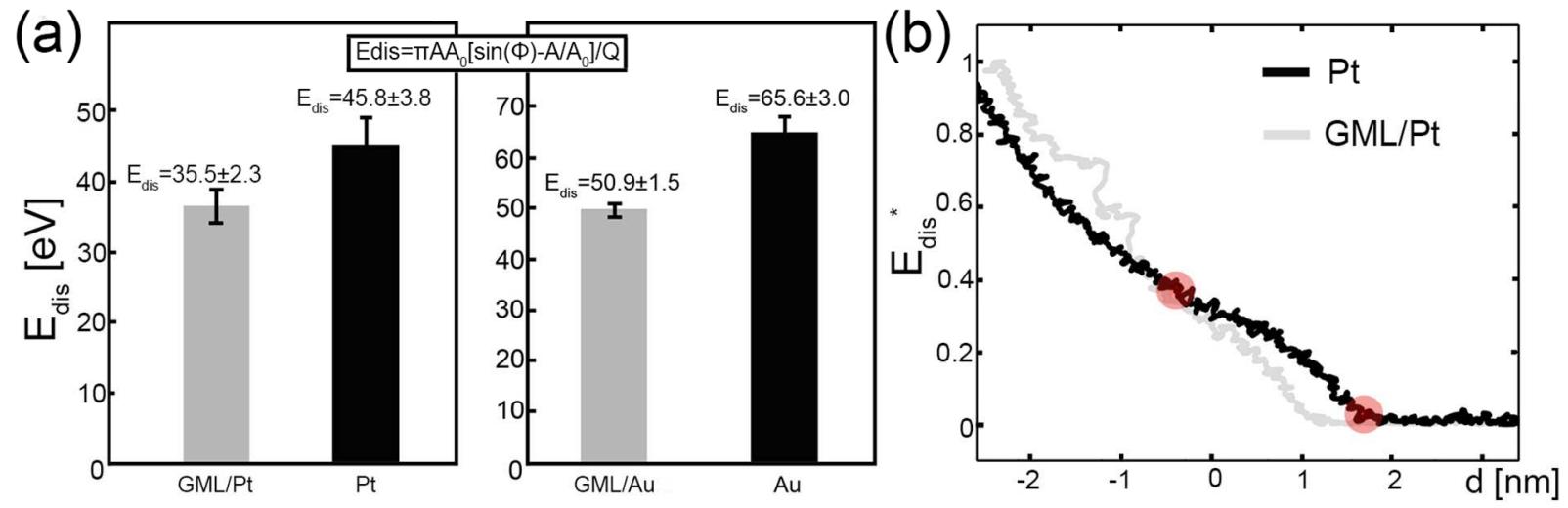

Figure 5: tip/surface dissipative interactions by means of AFM. (a) Dissipative interactions according to Cleveland's equation. ${ }^{41}$ Energy dissipation for GML on metal substrate and bare metal. Data on the left hand side and right hand side refer to Pt system and Au system respectively. (b) Normalized energy dissipation profile of Pt system. Black line represents for bare Pt and gray line represents for GML/Pt. Red circles represent inflection points related to the presence of capillary phenomena.

\section{Conclusions}

Summarizing, from SCA analysis obtained at the macro- and micro-range, it is possible to observe that only one layer of graphene changes the wetting properties of the surface, leading to different contact angles. It is well recognized that the wetting of a surface is related to its chemical and morphological properties. ${ }^{52,53}$ From AFM observations, the GML does not significantly change the roughness of the sample, thus the presence of GML dictates a different chemical state of the surface when interacting with water. This aspect highlights the low influence of the substrate and it agrees with non-transparency theory. ${ }^{22}$ Furthermore, nanoscale parameters (i.e. $\Delta \mathrm{dF}_{\mathrm{AD}}$ and $\mathrm{E}_{\mathrm{dis}}$ ) are able to capture the different interactions felt by the tip when 
oscillating in the proximity of GML/metal surface. The differences found in these two parameters demonstrate that interactions on bare metals are dominated by stronger capillary interactions at larger distances than on GML/metals, thus indicating different wetting behavior on coated and uncoated metals. The shorter conservative interactions when the tip is interacting with GML/metal systems compared to the bare metal are due to the presence of the graphene itself and its influence in shielding the surface/tip interaction. In conclusion, coherent evidences are provided at different length scales, which indicate the non-wetting transparency of graphene exposed to environmental conditions. The insights offered in this work will assist researchers when developing graphene-based applications in which interaction with water is the most important phenomenon, such as separation membranes.

\section{Acknowledgments}

A.V. and M. J. E. acknowledge financial support by the Ministerio de Economía y Competitividad (MINECO), Spain, through project MAT2012-38319-C02-01.

Electronic Supplementary Information (ESI) available: AFM images of metal substrates, the FFT from the AFM images and contact angles (static and dynamic) of surfaces. See DOI: 10.1039/b000000x

\section{References}

1. K. S. Novoselov, A. K. Geim, S. V. Morozov, D. Jiang, Y. Zhang, S. V. Dubonos, I. V. Grigorieva and A. A. Firsov, Science, 2004, 306, 666-669.

2. A. K. Geim, Science, 2009, 324, 1530-1534. 
3. A. H. C. Neto, F. Guinea, N. M. R. Peres, K. S. Novoselov and A. K. Geim, Reviews od modern physics, 2009, 81.

4. A. A. Balandin, Nat. Mater., 2011, 10, 569-581.

5. C. Lee, X. Wei, J. W. Kysar and J. Hone, Science, 2008, 321, 385-388.

6. C. Gómez-Navarro, M. Burghard and K. Kern, Nano Lett., 2008, 8, 2045-2049.

7. K. S. Kim, Y. Zhao, H. Jang, S. Y. Lee, J. M. Kim, K. S. Kim, J.-H. Ahn, P. Kim, J.-Y. Choi and B. H. Hong, Nature, 2009, 457, 706-710.

8. S. Bae, H. Kim, Y. Lee, X. Xu, J.-S. Park, Y. Zheng, J. Balakrishnan, T. Lei, H. Ri Kim, Y. I. Song, Y.-J. Kim, K. S. Kim, B. Ozyilmaz, J.-H. Ahn, B. H. Hong and S. Iijima, Nat. Nanotechnol., 2008, 5, 574-578.

9. K. S. Novoselov, V. I. Fal'ko, L. Colombo, P. R. Gellert, M. G. Schwab and K. Kim, Nature, 2012, 490, 192-200.

10. D. Cohen-Tanugi and J. C. Grossman, Nano Lett., 2012, 12, 3602-3608.

11. S. C. O'Hern, M. S. H. Boutilier, J.-C. Idrobo, Y. Song, J. Kong, T. Laoui, M. Atieh and R. Karnik, Nano Lett., 2014, 14, 1234-1241.

12. L. W. Drahushuk and M. S. Strano, Langmuir, 2012, 28, 16671-16678.

13. H. W. Kim, H. W. Yoon, S.-M. Yoon, B. M. Yoo, B. K. Ahn, Y. H. Cho, H. J. Shin, H. Yang, U. Paik, S. Kwon, J.-Y. Choi and H. B. Park, Science, 2013, 342, 91-95.

14. K. Celebi, J. Buchheim, R. M. Wyss, A. Droudian, P. Gasser, I. Shorubalko, J.-I. Kye, C. Lee and H. G. Park, Science, 2014, 344, 289-292.

15. S. Pan, R. Guo and W. Xu, Soft Matter, 2014, 10, 9187-9192.

16. S. Anandan, T. Narasinga Rao, M. Sathish, D. Rangappa, I. Honma and M. Miyauchi, ACS Applied Materials \& Interfaces, 2013, 5, 207-212.

17. L. Zhang, Z. Wang, C. Xu, Y. Li, J. Gao, W. Wang and Y. Liu, J. Mater. Chem., 2011, 21, 10399-10406.

18. V. K. Rana, M.-C. Choi, J.-Y. Kong, G. Y. Kim, M. J. Kim, S.-H. Kim, S. Mishra, R. P. Singh and C.-S. Ha, Macromol. Mater. Eng., 2011, 296, 131-140.

19. V. C. Sanchez, A. Jachak, R. H. Hurt and A. B. Kane, Chem. Res. Toxicol., 2011, 25, 1534.

20. Y. J. Shin, Y. Wang, H. Huang, G. Kalon, A. T. S. Wee, Z. Shen, C. S. Bhatia and H. Yang, Langmuir, 2010, 26, 3798-3802.

21. J. Rafiee, X. Mi, H. Gullapalli, A. V. Thomas, F. Yavari, Y. Shi, P. M. Ajayan and N. A. Koratkar, Nat. Mater., 2012, 11, 217-222.

22. R. Raj, S. C. Maroo and E. N. Wang, Nano Lett., 2013, 13, 1509-1515.

23. C.-J. Shih, Q. H. Wang, S. Lin, K.-C. Park, Z. Jin, M. S. Strano and D. Blankschtein, Phys. Rev. Lett., 2012, 109, 176101.

24. Zhiting Li, Yongjin Wang, Andrew Kozbial, Ganesh Shenoy, Feng Zhou, Rebecca McGinley, Patrick Ireland, Brittni Morganstein, Alyssa Kunkel, Sumedh P. Surwade, L. Li and H. Liu, Nature Material, 2013, 12, 925-031.

25. A. Kozbial, Z. Li, J. Sun, X. Gong, F. Zhou, Y. Wang, H. Xu, H. Liu and L. Li, Carbon, 2014, 74, 218-225.

26. M. E. Schrader, The Journal of Physical Chemistry, 1975, 79, 2508-2515.

27. Y.-H. Lu, C.-W. Yang and I.-S. Hwang, Langmuir, 2012, 28, 12691-12695.

28. C. A. Amadei, C.-Y. Lai, D. Heskes and M. Chiesa, The Journal of chemical physics, 2014, 141, -. 
29. A. Ashraf, Y. Wu, M. C. Wang, N. R. Aluru, S. A. Dastgheib and S. Nam, Langmuir, 2014, 30, 12827-12836.

30. T. Werder, J. H. Walther, R. L. Jaffe, T. Halicioglu and P. Koumoutsakos, The Journal of Physical Chemistry B, 2003, 107, 1345-1352.

31. F. Mugele, Nature, 2012, 11, 182-183.

32. S. Santos, C. A. Amadei, A. Verdaguer and M. Chiesa, The Journal of Physical Chemistry C, 2013, 117, 10615-10622.

33. B. Hammer and J. K. Norskov, Nature, 1995, 376, 238-240.

34. P. S. Skell and J. J. Havel, J. Am. Chem. Soc., 1971, 93, 6687-6688.

35. K. W. Bewig and W. A. Zisman, The Journal of Physical Chemistry, 1965, 69, 42384242.

36. A. J. Katan, M. H. Van Es and T. H. Oosterkamp, Nanotechnology, 2009, 20, 165703.

37. F. J. Giessibl, Physical Review B, 1997, 56, 16010-16015.

38. J. E. Sader and S. P. Jarvis, Appl. Phys. Lett., 2004, 84, 1801-1803.

39. S. Santos, L. Guang, T. Souier, K. Gadelrab, M. Chiesa and N. H. Thomson, Review of Scientific Instruments, 2012, 83, 043707.

40. R. García and A. San Paulo, Physical Review B, 1999, 60, 4961-4967.

41. J. P. Cleveland, B. Anczykowski, A. E. Schmid and V. B. Elings, Appl. Phys. Lett., 1998, 72, 2613-2615.

42. N. Wilson, A. Marsden, M. Saghir, C. Bromley, R. Schaub, G. Costantini, T. White, C. Partridge, A. Barinov, P. Dudin, A. Sanchez, J. Mudd, M. Walker and G. Bell, Nano Res., 2013, 6, 99-112.

43. A. Reina, X. Jia, J. Ho, D. Nezich, H. Son, V. Bulovic, M. S. Dresselhaus and J. Kong, Nano Lett., 2008, 9, 30-35.

44. L. Gao, W. Ren, H. Xu, L. Jin, Z. Wang, T. Ma, L.-P. Ma, Z. Zhang, Q. Fu, L.-M. Peng, X. Bao and H.-M. Cheng, Nat Commun, 2012, 3, 699.

45. O. Frank, J. Vejpravova, V. Holy, L. Kavan and M. Kalbac, Carbon, 2014, 68, 440-451.

46. M. H. Gass, U. Bangert, A. L. Bleloch, P. Wang, R. R. Nair and A. K. Geim, Nat Nano, 2008, 3, 676-681.

47. M. Brugnara, C. D. Volpe, S. Siboni and D. Zeni, Scanning, 2006, 28, 267-273.

48. N. A. Stelmashenko, J. P. Craven, A. M. Donald, E. M. Terentjev and B. L. Thiel, Journal of Microscopy, 2001, 204, 172-183.

49. John E Sader, Takayuki Uchihashi, Michael J Higgins, Alan Farrell, Yoshikazu Nakayama and S. P. Jarvis, Nanotechnology, 2005, 94-101.

50. C.-Y. Lai, T.-C. Tang, C. A. Amadei, A. J. Marsden, A. Verdaguer, N. Wilson and M. Chiesa, Carbon, 2014, 80, 784-792.

51. L. Zitzler, S. Herminghaus and F. Mugele, Physical Review B, 2002, 66, 155436.

52. J. N. Isreaelchvili, Intermolecular and surface forces Elsevier, Waltham MA (USA), 2011.

53. A. Méndez-Vilas, A. B. Jódar-Reyes and M. L. González-Martín, Small, 2009, 5, 13661390. 